\title{
Cubic Graphs Have Bounded Slope Parameter
}

\author{
Balázs Keszegh ${ }^{1,5}$, János Pach ${ }^{2,4,5}$, Dömötör Pálvölgyi ${ }^{3,4}$, and Géza Tóth ${ }^{5}$ \\ ${ }^{1}$ Central European University, Budapest \\ ${ }^{2}$ City College, CUNY, New York \\ ${ }^{3}$ Eötvös University, Budapest \\ ${ }^{4}$ Ecole Polytechnique Fédérale de Lausanne \\ ${ }^{5}$ A. Rényi Institute of Mathematics, Budapest
}

\begin{abstract}
We show that every finite connected graph $G$ with maximum degree three and with at least one vertex of degree smaller than three has a straight-line drawing in the plane satisfying the following conditions. No three vertices are collinear, and a pair of vertices form an edge in $G$ if and only if the segment connecting them is parallel to one of the sides of a previously fixed regular pentagon. It is also proved that every finite graph with maximum degree three permits a straight-line drawing with the above properties using only at most seven different edge slopes.
\end{abstract}

\section{Introduction}

A drawing of a graph $G$ is a representation of its vertices by distinct points in the plane and the edges by continuous arcs connecting the corresponding endpoints, not passing through any other point corresponding to a vertex. In a straight-line drawing [8], the edges are represented by (possibly crossing) segments. If it leads to no confusion, we make no notational or terminological distinction between the vertices (edges) of $G$ and the points (arcs) representing them.

There are several widely known parameters of graphs measuring how far $G$ is from being planar. For instance, the thickness of $G$ is the smallest number of its planar subgraphs whose union is $G$ [14]. The geometric thickness of $G$ is the smallest number of crossing-free subgraphs of a straight-line drawing of $G$, whose union is $G$ [11]. The slope number of $G$ is the minimum number of distinct edge slopes in a straightline drawing of $G[16]$. It follows directly from the definitions that the thickness of any graph is at most as large as its geometric thickness, which, in turn, cannot exceed its slope number. For many interesting results about these parameters, consult

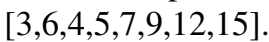

The slope parameter of a graph was defined by Ambrus, Barát, and P. Hajnal [1], as follows. By abusing the usual terminology, we say that the slope of a line $\ell$ in the $x y$ plane is the smallest angle $\alpha \in[0, \pi)$ such that $\ell$ can be rotated into a position parallel to the $x$-axis by a clockwise turn through $\alpha$. Given a set of points $P$ in the plane and a set of slopes $\Sigma$, define $G(P, \Sigma)$ as the graph on the vertex set $P$, in which two vertices

\footnotetext{
* Research supported by grants from NSF, NSA, PSC-CUNY and the Hungarian Research Foundation OTKA.
} 
$p, q \in P$ are connected by an edge if and only if the slope of the line $p q$ belongs to $\Sigma$. The slope parameter $s(G)$ of $G$ is the size of the smallest set of slopes $\Sigma$ such that $G$ is isomorphic to $G(P, \Sigma)$ for a suitable set of points $P$ in the plane. This definition was motivated by the fact that all connections (edges) in an electrical circuit (graph) $G$ can be easily realized by the overlay of $s(G)$ finely striped electrically conductive layers.

The slope parameter, $s(G)$, is closely related to the three other graph parameters mentioned before. For instance, for triangle-free graphs, $s(G)$ is at least as large as the slope number of $G$, the largest of the three quantities above. On the other hand, it sharply differs from them in the sense that the slope parameter of a complete graph on $n$ vertices is one, while the thickness, the geometric thickness, and the slope number of $K_{n}$ tend to infinity as $n \rightarrow \infty$. Jamison [10] proved that the slope number of $K_{n}$ is $n$.

Any graph $G$ of maximum degree two splits into vertex disjoint cycles, paths, and possibly isolated vertices. Hence, for such graphs we have $s(G) \leq 3$. In contrast, as was shown by Barát et al. [2], for any $d \geq 5$, there exist graphs of maximum degree $d$, whose slope parameters are arbitrarily large.

A graph is said to be cubic if the degree of each of its vertices is at most three. A cubic graph is subcubic if each of its connected components has a vertex of degree smaller than three.

The aim of this note is to prove

Theorem 1. Every cubic graph has slope parameter at most seven.

We will refer to the angles $i \pi / 5,0 \leq i \leq 4$, as the five basic slopes. In Sect. 2, we prove the following statement, which constitutes the first step of the proof of Theorem 1.

Theorem 2. Every subcubic graph has slope parameter at most five. Moreover, this can be realized by a straight-line drawing such that no three vertices are on a line and each edge has one of the five basic slopes.

Using the fact that in the drawing guaranteed by Theorem 2 no three vertices are collinear, we can also conclude that the slope number of every subcubic graph is at most five. In [12], however, it was shown that this number is at most four and for cubic graphs it is at most five. This was improved for connected cubic graphs in [13] to four.

\section{Proof of Theorem 2}

The proof is by induction on the number of vertices of the graph. Clearly, the statement holds for graphs with fewer than three vertices. Let $n$ be fixed and suppose that we have already established the statement for graphs with fewer than $n$ vertices. Let $G$ be a subcubic graph of $n$ vertices. We can assume that $G$ is connected, otherwise we can draw each of its connected components separately and translate the resulting drawings through suitable vectors.

To obtain a drawing of $G$, we have to find proper locations for its vertices. At each inductive step, we start with a drawing of a subgraph of $G$ satisfying the conditions and extend it by adding a vertex. At a given stage of the procedure, for any vertex $v$ that has already been added, consider the (basic) slopes of all edges adjacent to $v$ that have already been drawn, and let $\operatorname{sl}(v)$ denote the set of integers $0 \leq i<5 \bmod 5$ 
for which $i \pi / 5$ is such a slope. That is, at the beginning $\operatorname{sl}(v)$ is undefined, then it gets defined, and later it may change (expand). Analogously, for any edge $u v$ of $G$, denote by $\operatorname{sl}(u v)$ the integer $0 \leq i<5 \bmod 5$ for which the slope of $u v$ is $i \pi / 5$.

Case 1: $G$ has a vertex of degree one. Assume without loss of generality, that $v$ is a vertex of degree one, and let $w$ denote its only neighbor. Deleting $v$ from $G$, the degree of $w$ in the resulting graph $G^{\prime}$ is at most two. Therefore, by the induction hypothesis, $G^{\prime}$ has a drawing meeting the requirements. As $w$ has degree at most $t w o$, there is a basic slope $\sigma$ such that no other vertex of $G^{\prime}$ lies on the line $\ell$ of slope $\sigma$ that passes through $w$. Draw all five lines of basic slopes through each vertex of $G^{\prime}$. These lines intersect $\ell$ in finitely many points. We can place $v$ at any other point of $\ell$, to obtain a proper drawing of $G$.

From now on, assume that $G$ has no vertex of degree one.

Case 2: $G$ has no cycle that passes through a vertex of degree two. Since $G$ is subcubic, it contains a vertex $w$ of degree two such that $G$ is the union of two graphs, $G_{1}$ and $G_{2}$, having only vertex $w$ in common. Both $G_{1}$ and $G_{2}$ are subcubic and have fewer than $n$ vertices, so by the induction hypothesis both of them have a drawing satisfying the conditions. Translate the drawing of $G_{2}$ so that the points representing $w$ in the two drawings coincide. Since $w$ has degree one in both $G_{1}$ and $G_{2}$, by a possible rotation of $G_{2}$ about $w$ through an angle that is a multiple of $\pi / 5$, we can achieve that the two edges adjacent to $w$ are not parallel. By scaling $G_{2}$ from $w$, if necessary, we can also achieve that the slope of no segment between a vertex of $G_{1} \backslash w$ and a vertex of $G_{2} \backslash w$ is a basic slope. Thus, the resulting drawing of $G$ meets the requirements.

Case 3: $G$ has a cycle passing through a vertex of degree two. If $G$ itself is a cycle, we can easily draw it. If it is not the case, let $C$ be a shortest cycle which contains a vertex of degree two. Let $u_{0}, u_{1}, \ldots, u_{k}$ denote the vertices of $C$, in this order, such that $u_{0}$ has degree two and $u_{1}$ has degree three. The indices are understood $\bmod k+1$, that is, for instance, $u_{k+1}=u_{0}$. It follows from the minimality of $C$ that $u_{i}$ and $u_{j}$ are not connected by an edge of $G$ whenever $|i-j|>1$.

Since $G \backslash C$ is subcubic, by assumption, it permits a straight-line drawing satisfying the conditions. Each $u_{i}$ has at most one neighbor in $G \backslash C$. Denote this neighbor by $t_{i}$, if it exists. For every $i$ for which $t_{i}$ exists, we place $u_{i}$ on a line passing through $t_{i}$. We place the $u_{i}$ 's one by one, "very far" from $G \backslash C$, starting with $u_{1}$. Finally, we arrive at $u_{0}$, which has no neighbor in $G \backslash C$, so that it can be placed at the intersection of two lines of basic slope, through $u_{1}$ and $u_{k}$, respectively. We have to argue that our method does not create "unnecessary" edges, that is, we never place two independent vertices in such a way that the slope of the segment connecting them is a basic slope. In what follows, we make this argument precise.

We determine the locations of $u_{0}, u_{1}, \ldots, u_{k}$ by using the below described PROCE$\operatorname{DURE}\left(G, C, u_{0}, u_{1}, x\right)$, where $G$ is our subcubic graph, $C$ is the shortest cycle passing through a vertex of degree two, $u_{0}$ is such a vertex, $u_{1}$ is a neighbor of $u_{0}$ on $C$, whose degree is three, and $x$ is a real parameter. Note that $\operatorname{Procedure}\left(G, C, u_{0}, u_{1}, x\right)$ is a nondeterministic algorithm, as we have more than one choice at certain steps. (However, it is very easy to make it deterministic.) 

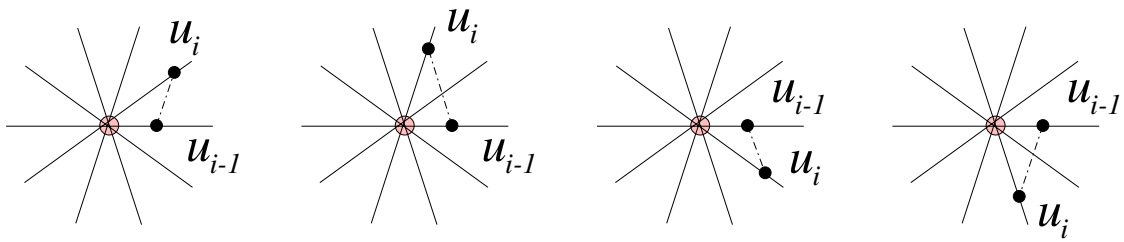

Fig. 1. The four possible locations of $u_{i}$

$\operatorname{Procedure}\left(G, C, u_{0}, u_{1}, x\right)$

- Step 0. Since $G \backslash C$ is subcubic, it has a representation with the five basic slopes. Take such a representation, scaled and translated in such a way that $t_{1}$ (which exists since the degree of $u_{1}$ is three) is at the origin, and all other vertices are within unit distance from it.

For any $i, 2 \leq i \leq k$, for which $u_{i}$ does not have a neighbor in $G \backslash C$, let $t_{i}$ be any unoccupied point closer to the origin than 1 , such that the slope of none of the lines connecting $t_{i}$ to $t_{1}, t_{2}, \ldots t_{i-1}$ or to any other already embedded point of $G \backslash C$ is a basic slope.

For any point $p$ and for any $i \bmod 5$, let $\ell_{i}(p)$ denote the line with $i$ th basic slope, $i \pi / 5$, passing through $p$. Let $\ell_{i}$ stand for $\ell_{i}(O)$, where $O$ denotes the origin.

We will place $u_{1}, \ldots, u_{k}$ recursively, so that $u_{j}$ is placed on $\ell_{i}\left(t_{j}\right)$, for a suitable $i$. Once the position of $u_{j}$ has already been fixed on some $\ell_{i}\left(t_{j}\right)$, define $i n d\left(u_{j}\right)$, the index of $u_{j}$, to be $i$. (Again, the indices are taken mod 5. Thus, for example, $\left|i-i^{\prime}\right| \geq 2$ is equivalent to saying that $i \neq i^{\prime}$ and $i \neq i^{\prime} \pm 1 \bmod 5$.) Start with $u_{1}$. The degree of $t_{1}$ in $G \backslash C$ is at most $t w o$, so that at the beginning the set $s l\left(t_{1}\right)$ (defined in the first paragraph of this section) has at most two elements. Let $l \notin s l\left(t_{1}\right)$. Direct the line $\ell_{l}\left(t_{1}\right)$ arbitrarily, and place $u_{1}$ on it at distance $x$ from $t_{1}$ in the positive direction. (According to this rule, if $x<0$, then $u_{1}$ is placed on $\ell_{l}\left(t_{1}\right)$ at distance $|x|$ from $t_{1}$ in the negative direction.)

Suppose that $u_{1}, u_{2}, \ldots, u_{i-1}$ have been already placed and that $u_{i-1}$ lies on the line $\ell_{l}\left(t_{i-1}\right)$, that is, we have $\operatorname{ind}\left(u_{i-1}\right)=l$.

- STEP $i$. We place $u_{i}$ at one of the following four locations (see Fig. 1):

(1) the intersection of $\ell_{l+1}\left(t_{i}\right)$ and $\ell_{l+2}\left(u_{i-1}\right)$;

(2) the intersection of $\ell_{l+2}\left(t_{i}\right)$ and $\ell_{l+3}\left(u_{i-1}\right)$;

(3) the intersection of $\ell_{l-1}\left(t_{i}\right)$ and $\ell_{l-2}\left(u_{i-1}\right)$;

(4) the intersection of $\ell_{l-2}\left(t_{i}\right)$ and $\ell_{l-3}\left(u_{i-1}\right)$.

Choose from the above four possibilities so that the edge $u_{i} t_{i}$ is not parallel to any other edge already drawn and adjacent to $t_{i}$, i.e., before adding the edge $u_{i} t_{i}$ to the drawing, $s l\left(t_{i}\right)$ did not include $s l\left(u_{i} t_{i}\right)$.

It follows directly from (1)-(4) that the edge $u_{i} u_{i-1}$ is not parallel to any other edge already drawn and adjacent to $u_{i-1}$. That is, before adding the edge $u_{i} u_{i-1}$ to the drawing, we had $s l\left(u_{i} u_{i-1}\right) \notin s l\left(u_{i-1}\right)$. Avoiding for $u_{i} t_{i}$ the slopes of the edges 
already incident to $t_{i}$, leaves available two of the choices (1), (2), (3), (4). Some simple geometric calculations show that, for any possible location of $u_{i}$, we have

$1.6 \overline{O u_{i-1}}-4<2 \cos \left(\frac{\pi}{5}\right) \overline{O u_{i-1}}-4<\overline{O u_{i}}<2 \cos \left(\frac{\pi}{5}\right) \overline{O u_{i-1}}+4<1.7 \overline{O u_{i-1}}+4$.

Thus, if $|x| \geq 50$, then we obtain by induction that

$$
1.5 \overline{O u_{i-1}}<\overline{O u_{i}} .
$$

Here, we used that $x-1<\overline{O u_{1}}$ and that, by the induction hypothesis, $\overline{O u_{j}}$ is strictly increasing for $j<i$, therefore, we have $x-1<\overline{O u_{i-1}}$.

We have to verify that the above procedure does not produce "unnecessary" edges, that is, the following statement is true.

Claim 1. Suppose that $|x| \geq 50$.

(i) The slope of $u_{i} u_{j}$ is not a basic slope, for any $j<i-1$.

(ii) The slope of $u_{i} v$ is not a basic slope, for any $v \in V(G \backslash C)$.

Proof. (i) Suppose that the slope of $u_{i} u_{j}$ is a basic slope for some $j<i-1$. By repeated application of inequality (1), we obtain that $\overline{O u_{i}}>1.5^{i-j} \overline{O u_{j}}>2 \overline{O u_{j}}$. On the other hand, if $u_{i} u_{j}$ has a basic slope, then easy geometric calculations show that $\overline{O u_{i}}<2 \cos \left(\frac{\pi}{5}\right) \overline{O u_{j}}+4<2 \overline{O u_{j}}$, a contradiction.

(ii) Suppose for simplicity that $t_{i} u_{i}$ has slope 0, i.e., it is horizontal. By the construction, no vertex $v$ of $G \backslash C$ determines a horizontal segment with $t_{i}$, but all of them are within distance 2 from $t_{i}$. As $\overline{O u_{i}}>x-1$, segment $v u_{i}$ is almost, but not exactly horizontal. That is, we have $0<\left|\angle t_{i} u_{i} v\right|<\pi / 5$, contradiction.

Suppose that STEP $0, \mathrm{STEP} 1, \ldots, \mathrm{STEP} k$ have already been completed. It remains to determine the position of $u_{0}$. We need some preparation.

Claim 2. There exist two integers $0 \leq l, l^{\prime}<5$ with $\left|l-l^{\prime}\right| \geq 2 \bmod 5$ such that starting the PROCEDURE with ind $\left(u_{1}\right)=l$ and with ind $\left(u_{1}\right)=l^{\prime}$, we can continue so that ind $\left(u_{2}\right)$ is the same.

Proof. Suppose that the degrees of $t_{1}$ and $t_{2}$ in $G \backslash C$ are $t w o$, that is, there are two forbidden lines for both $u_{1}$ and $u_{2}$. In the other cases, when the degree of $t_{1}$ or the degree of $t_{2}$ is less than $t w o$, or when $t_{1}=t_{2}$, the proof is similar, but simpler. We can place $u_{1}$ on $\ell_{l}\left(t_{1}\right)$ for any $l \notin s l\left(t_{1}\right)$. Therefore, we have three choices, two of which, $\ell_{\alpha}\left(t_{1}\right)$ and $\ell_{\beta}\left(t_{1}\right)$, are not consecutive, so that $|\alpha-\beta| \geq 2$.

The vertex $u_{2}$ cannot be placed on $\ell_{m}\left(t_{2}\right)$ for any $m \in \operatorname{sl}\left(t_{2}\right)$, so there are three possible lines for $u_{2}: \ell_{x}\left(t_{2}\right), \ell_{y}\left(t_{2}\right), \ell_{z}\left(t_{2}\right)$, say. For any fixed location of $u_{1}$, we can place $u_{2}$ on at least two of the lines $\ell_{x}\left(t_{2}\right), \ell_{y}\left(t_{2}\right)$, and $\ell_{z}\left(t_{2}\right)$. Therefore, at least one of them, $\ell_{x}\left(t_{2}\right)$, say, can be used for both locations of $u_{1}$.

Claim 3. We can place the vertices $u_{1}, u_{2}, \ldots, u_{k}$ using the PROCEDURE so that $\left|\operatorname{ind}\left(u_{1}\right)-\operatorname{ind}\left(u_{k}\right)\right| \geq 2 \bmod 5$.

Proof. By Claim 2, there are two placements of the vertices of $C \backslash\left\{u_{0}, u_{k}\right\}$, denoted by $u_{1}, u_{2}, \ldots, u_{k-1}$ and by $u_{1}^{\prime}, u_{2}^{\prime}, \ldots, u_{k-1}^{\prime}$ such that $\left|i n d\left(u_{1}\right)-i n d\left(u_{1}^{\prime}\right)\right| \geq 2 \bmod 5$, and $i n d\left(u_{i}\right)=\operatorname{ind}\left(u_{i}^{\prime}\right)$ for all $i \geq 2$. That is, we can start placing the vertices on 

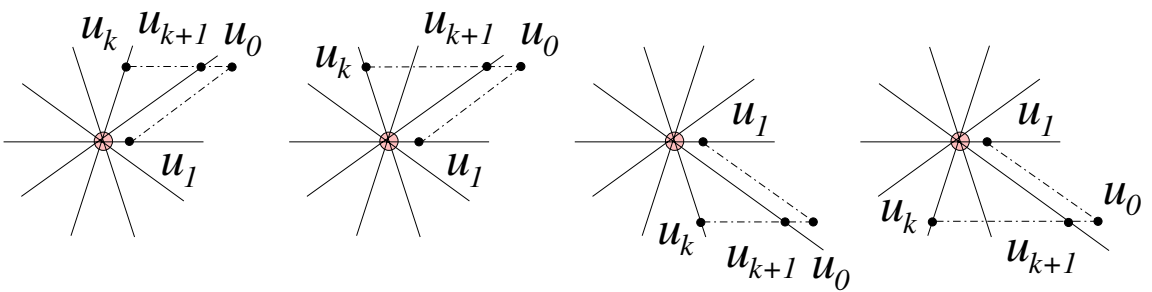

Fig. 2. The four possible locations of $u_{0}$

two nonneighboring lines so that from the second step of the PROCEDURE we use the same lines. We show that we can place $u_{k}$ such that $u_{1}$ and $u_{k}$, or $u_{1}^{\prime}$ and $u_{k}$ are on nonneighboring lines. Having placed $u_{k-1}$ ( or $u_{k-1}^{\prime}$ ), we have four choices for $i n d\left(u_{k}\right)$. Two of them can be ruled out by the condition $i n d\left(u_{k}\right) \notin \operatorname{sl}\left(t_{k}\right)$. We still have two choices. Since $u_{1}$ and $u_{1}^{\prime}$ are on nonneighboring lines, there is only one line which is neighboring of both of them. Therefore, we still have at least one choice for ind $\left(u_{k}\right)$ such that $\left|\operatorname{ind}\left(u_{1}\right)-i n d\left(u_{k}\right)\right| \geq 2$ or $\left|\operatorname{ind}\left(u_{1}^{\prime}\right)-i n d\left(u_{k}\right)\right| \geq 2$.

- STEP $k+1$. Let $i=i n d\left(u_{1}\right), j=i n d\left(u_{k}\right)$, and assume, by Claim 3 , that $|i-j| \geq 2$ mod 5 . Consider the lines $\ell_{i-1}\left(u_{1}\right)$ and $\ell_{i+1}\left(u_{1}\right)$. One of them, $\ell_{i+1}\left(u_{1}\right)$, say, does not separate the vertices of $G \backslash C$ from $u_{k}$, the other one does.

Place $u_{0}$ at the intersection of $\ell_{i+1}\left(u_{1}\right)$ and $\ell_{i}\left(u_{k}\right)$.

Claim 4. Suppose that $|x| \geq 50$.

(i) The slope of $u_{0} u_{j}$ is not a basic slope, for any $1<j<k$.

(ii) The slope of $u_{0} v$ is not a basic slope, for any $v \in V(G \backslash C)$.

Proof. (i) Denote by $u_{k+1}$ the intersection of $\ell_{i+1}(O)$ and $\ell_{i}\left(u_{k}\right)$. Suppose that the slope of $u_{0} u_{j}$ is a basic slope for some $1<j<k$. As in the proof of Claim 1 , by repeated application of inequality 1 , we obtain that $\overline{O u_{k+1}}>1.5^{k+1-j} \overline{O u_{j}}>2 \overline{O u_{j}}$. On the other hand, by an easy geometric argument, if the slope of $u_{0} u_{j}$ is a basic slope, then $\overline{O u_{k+1}}<2 \cos \left(\frac{\pi}{5}\right) \overline{O u_{j}}+4<2 \overline{O u_{j}}$, a contradiction, provided that $|x| \geq 50$.

(ii) For any vertex $v \in G \backslash C$, the slope of the segment $u_{0} v$ is strictly between $i \pi / 5$ and $(i+1) \pi / 5$, therefore, it is not a basic slope. See Fig. 2. This concludes the proof of the claim and hence Theorem 2 .

\section{Proof of Theorem 1}

First we note that if $G$ is connected, then Theorem 1 is an easy corollary to Theorem 2 . Indeed, delete any vertex, and then put it back using two extra directions. If $G$ is not connected, the only problem that may arise is that these extra directions can differ for different components. We will define a family of drawings for each component of $G$, depending on a parameter $\varepsilon$, and then choose the values of these parameters in such a way that the extra directions will coincide.

Suppose that $G$ is a cubic graph. If a connected component is not 3-regular then, by Theorem 2, it can be drawn using the five basic slopes. If a connected component is a 
complete graph $K_{4}$ on four vertices, then it can also be drawn using the basic slopes. For the sake of simplicity, suppose that we do not have such components, ie. each connected component $G^{1}, \ldots, G^{m}$ of $G$ is 3-regular and none of them is isomorphic to $K_{4}$.

First we concentrate on $G^{1}$. Let $C$ be a shortest cycle in $G^{1}$. We distinguish two cases.

Case 1: $C$ is not a triangle. Denote by $u_{0}, \ldots, u_{k}$ the vertices of $C$, and let $t_{0}$ be the neighbor of $u_{0}$ not belonging to $C$. Delete the edge $u_{0} t_{0}$, and let $\bar{G}$ be the resulting graph.

Case 2: $C$ is a triangle. Then every vertex of $C$ has precisely one neighbor that does not belong to $C$. If all these neighbors coincide, then $G^{1}$ is a complete graph on four vertices, contradicting our assumption. So one vertex of $C, u_{0}$, say, has a neighbor $t_{0}$ which does not belong to $C$ and which is not adjacent to the other two vertices, $u_{1}$ and $u_{2}$, of $C$. Delete the edge $u_{0} t_{0}$, and let $\bar{G}$ be the resulting graph.

Observe that in both cases, $u_{k}$ and $t_{0}$ are not connected in $G^{1}$. Indeed, suppose for a contradiction that they are connected. In the first case, $G^{1}$ would contain the triangle $u_{0} u_{k} t_{0}$, contradicting the minimality of $C$. In the second case, the choice of $u_{0}$ would be violated.

There will be exactly two edges with extra directions, $u_{0} u_{1}$ and $u_{0} t_{0}$. The slope of $u_{0} u_{1}$ will be very close to a basic slope and the slope of $u_{0} t_{0}$ will be decided at the end, but we will show that almost any choice will do.

For any real $x$ and $\varepsilon>0$, define ModifiedProcedure $\left(\bar{G}, C, u_{0}, u_{1}, x, \varepsilon\right)$, as follows. Let STEPS $0,1, \ldots, k$ be identical to the corresponding STEPS of PROCEDU$\operatorname{RE}\left(\bar{G}, C, u_{0}, u_{1}, x\right)$.

- STEP $k+1$. If there is a segment, determined by the vertices of $G \backslash C$, of slope $i \pi / 5+\varepsilon$ or $i \pi / 5-\varepsilon$, for any $0 \leq i<5$, then STOP. In this case, we say that $\varepsilon$ is 1 -bad for $\bar{G}$.

Otherwise, when $\varepsilon$ is $1-\operatorname{good}$, let $i=i n d\left(u_{1}\right)$ and $j=i n d\left(u_{k}\right)$. We can assume that $|i-j| \geq 2 \bmod 5$. Consider the lines $\ell_{i-1}\left(u_{1}\right)$ and $\ell_{i+1}\left(u_{1}\right)$. One of them does not separate the vertices of $G \backslash C$ from $u_{k}$, the other one does.

If $\ell_{i-1}\left(u_{1}\right)$ separates $G \backslash C$ from $u_{k}$, then place $u_{0}$ at the intersection of $\ell_{i+1}\left(u_{1}\right)$ and the line through $u_{k}$ with slope $i \pi / 5+\varepsilon$. If $\ell_{i+1}\left(u_{1}\right)$ separates $G \backslash C$ from $u_{k}$, then place $u_{0}$ at the intersection of $\ell_{i-1}\left(u_{1}\right)$ and the line through $u_{k}$ with slope $i \pi / 5-\varepsilon$.

Since Steps $0, \ldots, k$ are identical in ModifiedProcedure $\left(\bar{G}, C, u_{0}, u_{1}, x, \varepsilon\right)$ and in Procedure $\left(\bar{G}, C, u_{0}, u_{1}, x\right)$, the Claims 1, 2, and 3 also hold for the ModifiedPROCEDURE.

Moreover, it is easy to see that the analogue of Claim 4 also holds with an identical proof, provided that $\varepsilon$ is sufficiently small: $0<\varepsilon<1 / 100$.

Claim 4'. Suppose that $|x| \geq 50$ and $0<\varepsilon<1 / 100$.

(i) The slope of $u_{0} u_{j}$ is not a basic slope, for any $1<j<k$.

(ii) The slope of $u_{0} v$ is not a basic slope, for any $v \in V(\bar{G} \backslash C)$.

Perform ModifiedProcedure $\left(\bar{G}, C, u_{0}, u_{1}, x, \varepsilon\right)$ for a fixed $\varepsilon$, and observe how the drawing changes as $x$ varies. For any vertex $u_{i}$ of $C$, let $u_{i}(x)$ denote the position of 
$u_{i}$, as a function of $x$. For every $i$, the function $u_{i}(x)$ is linear, that is, $u_{i}$ moves along a line as $x$ varies.

Claim 5. If $\varepsilon$ is 1-good, then with finitely many exceptions, for every value of $x$, MODIFiedProcedure $\left(\bar{G}, C, u_{0}, u_{1}, x, \varepsilon\right)$ produces a proper drawing of $\bar{G}$.

Proof. Claims 1, 2, 3, and 4' imply Claim 5 for $|x| \geq 50$. Let $u$ and $v$ be two vertices of $\bar{G}$. Since $u(x)$ and $v(x)$ are linear functions, their difference, $\boldsymbol{u v}(x)$, is also linear.

If $u v$ is an edge of $\bar{G}$, then the direction of $\boldsymbol{u v}(x)$ is the same for all $|x| \geq 50$. Therefore, it is the same for all values of $x$, with the possible exception of one value, for which $\boldsymbol{u} \boldsymbol{v}(x)=0$ holds.

If $u v$ is not an edge of $\bar{G}$, then the slope of $\boldsymbol{u} \boldsymbol{v}(x)$ is not a basic slope for any $|x| \geq 50$. Therefore, with the exception of at most five values of $x$, the slope of $\boldsymbol{u} \boldsymbol{v}(x)$ is never a basic slope, nor does $\boldsymbol{u v}(x)=0$ hold.

Take a closer look at the relative position of the endpoints of the missing edge, $u_{0}(x)$ and $t_{0}(x)$. Since $t_{0} \in \bar{G} \backslash C, t_{0}=t_{0}(x)$ is the same for all values of $x$. The position of $u_{0}=u_{0}(x)$ is a linear function of $x$. Let $\ell$ be the line determined by the function $u_{0}(x)$. If $\ell$ passes through $t_{0}$, then we say that $\varepsilon$ is $2-b a d$ for $\bar{G}$. If $\varepsilon$ is 1 -good and it is not 2-bad for $\bar{G}$, then we say that it is 2 -good for $\bar{G}$. If $\varepsilon$ is 2 -good, then by varying $x$ we can achieve almost any slope for the edge $t_{0} u_{0}$. This will turn out to be crucially important, because we want to attain that these slopes coincide in all components.

Claim 6. Suppose that the values $0<\varepsilon, \delta<1 / 100$ are 1 -good for $\bar{G}$. Then at least one of them is 2-good for $\bar{G}$.

Proof. Suppose, for simplicity, that $i n d\left(u_{1}\right)=0, \operatorname{ind}\left(u_{k}\right)=2$, and that $u_{1}$ and $u_{k}$ are in the right half-plane (of the vertical line through the origin). The other cases can be settled analogously. To distinguish between ModifiedProcedure $\left(\bar{G}, C, u_{0}, u_{1}, x, \varepsilon\right)$ and ModifiedProcedure $\left(\bar{G}, C, u_{0}, u_{1}, x, \delta\right)$, let $u_{0}^{\varepsilon}(x)$ denote the position of $u_{0}$ obtained by the first procedure and $u_{0}^{\delta}(x)$ its position obtained by the second. Let $\ell^{\varepsilon}$ and $\ell^{\delta}$ denote the lines determined by the functions $u_{0}^{\varepsilon}(x)$ and $u_{0}^{\delta}(x)$. Suppose that $x$ is very large. Since, by (1), we have $\overline{u_{k}(x) O}>1.5 \overline{u_{1}(x) O}$, both $u_{0}^{\varepsilon}(x)$ and $u_{0}^{\delta}(x)$ are above the line $\ell_{\pi / 10}$. On the other hand, if $x<0$ is very small (i.e., if $|x|$ is very big), both $u_{0}^{\varepsilon}(x)$ and $u_{0}^{\delta}(x)$ lie below the line $\ell_{\pi / 10}$. It follows that the slopes of $\ell^{\varepsilon}$ and $\ell^{\delta}$ are larger than $\pi / 10$, but smaller than $\pi / 5$.

Suppose that neither $\varepsilon$ nor $\delta$ is 2 -good. Then both $\ell^{\varepsilon}$ and $\ell^{\delta}$ pass through $t_{0}$. That is, for a suitable value of $x$, we have $u_{0}^{\varepsilon}(x)=t_{0}$. We distinguish two cases.

Case 1: $u_{0}^{\varepsilon}(x)=t_{0}=u_{k}(x)$. Then, as $x$ varies, the line determined by $u_{k}(x)$ coincides with $\ell_{2}\left(t_{0}\right)$. Consequently, $t_{0}$ and $u_{k}$ are connected in $G^{1}$, a contradiction.

Case 2: $u_{0}^{\varepsilon}(x)=t_{0} \neq u_{k}(x)$. In order to get a contradiction, we try to determine the position of $u_{0}^{\delta}(x)$. Considering STEP $k+1$ in both ModifiedProcedure $\left(\bar{G}, C, u_{0}, u_{1}, x, \varepsilon\right)$ and in ModifiedProcedure $\left(\bar{G}, C, u_{0}, u_{1}, x, \delta\right)$, we can conclude that $u_{1}(x)$ lies on $\ell_{1}\left(t_{0}\right), u_{0}^{\delta}(x)$ lies on $\ell_{1}\left(u_{1}(x)\right)$, therefore, $u_{0}^{\delta}(x)$ lies on $\ell_{1}\left(t_{0}\right)$. On the other hand, $u_{0}^{\delta}(x)$ lies on $\ell^{\delta}$, and, by assumption, $\ell^{\delta}$ passes through $t_{0}$. However, we have shown that $\ell^{\delta}$ and $\ell_{1}\left(t_{0}\right)$ have different slopes, therefore, $u_{0}^{\delta}(x)$ must be at their intersection point, so we have $u_{0}^{\delta}(x)=u_{0}^{\varepsilon}(x)=t_{0}$. 
Considering again $\operatorname{SteP} k+1$ in $\operatorname{ModifiedProcedure}\left(\bar{G}, C, u_{0}, u_{1}, x, \varepsilon\right)$ and in ModifiedProcedure $\left(\bar{G}, C, u_{0}, u_{1}, x, \delta\right)$, we can conclude that the point $u_{0}^{\delta}(x)=$ $t_{0}=u_{0}^{\varepsilon}(x)$ belongs to both $\ell_{\varepsilon}\left(u_{k}(x)\right)$ and $\ell_{\delta}\left(u_{k}(x)\right)$. This contradicts our assumption that $u_{k}(x)$ is different from $u_{0}^{\delta}(x)=t_{0}=u_{0}^{\varepsilon}(x)$.

By Claim 5, for every $\varepsilon<1 / 100$ and with finitely many exceptions for every value of $x$, ModifiedProcedure $\left(\bar{G}, C, u_{0}, u_{1}, x, \varepsilon\right)$ produces a proper drawing of $\bar{G}$. When we want to add the edge $u_{0} t_{0}$, the slope of $u_{0}(x) t_{0}$ may coincide with the slope of $u(x) u^{\prime}(x)$, for some $u, u^{\prime} \in \bar{G}$. The following statement guarantees that this does not happen "too often". We use $\alpha(\boldsymbol{u})$ to denote the slope of a vector $\boldsymbol{u}$.

Claim 7. Let $\boldsymbol{u}(x)$ and $\boldsymbol{v}(x): R \rightarrow R^{2}$ be two linear functions, and let $\ell(u)$ and $\ell(v)$ denote the lines determined by $\boldsymbol{u}(x)$ and $\boldsymbol{v}(x)$. Suppose that for some $x_{1}<x_{2}<$ $x_{3}$, the vectors $\boldsymbol{u}, \boldsymbol{v}$ do not vanish and that their slopes coincide, that is, $\alpha\left(\boldsymbol{u}\left(x_{1}\right)\right)=$ $\alpha\left(\boldsymbol{v}\left(x_{1}\right)\right), \alpha\left(\boldsymbol{u}\left(x_{2}\right)\right)=\alpha\left(\boldsymbol{v}\left(x_{2}\right)\right)$, and $\alpha\left(\boldsymbol{u}\left(x_{3}\right)\right)=\alpha\left(\boldsymbol{v}\left(x_{3}\right)\right)$. Then $\ell(u)$ and $\ell(v)$ must be parallel.

Proof. If $\ell(u)$ passes through the origin, then for every value of $x, \boldsymbol{u}(x)$ has the same slope. In particular, $\alpha\left(\boldsymbol{v}\left(x_{1}\right)\right)=\alpha\left(\boldsymbol{v}\left(x_{2}\right)\right)=\alpha\left(\boldsymbol{v}\left(x_{3}\right)\right)$. Therefore, $\ell(v)$ also passes through the origin and is parallel to $\ell(u)$. (In fact, we have $\ell(u)=\ell(v)$.) We can argue analogously if $\ell(u)$ passes through the origin. Thus, in what follows, we can assume that neither $\ell(u)$ nor $\ell(v)$ passes through the origin.

Suppose that $\alpha\left(\boldsymbol{u}\left(x_{1}\right)\right)=\alpha\left(\boldsymbol{v}\left(x_{1}\right)\right), \alpha\left(\boldsymbol{u}\left(x_{2}\right)\right)=\alpha\left(\boldsymbol{v}\left(x_{2}\right)\right)$, and $\alpha\left(\boldsymbol{u}\left(x_{3}\right)\right)=$ $\alpha\left(\boldsymbol{v}\left(x_{3}\right)\right)$. For any $x$, define $\boldsymbol{w}(x)$ as the intersection point of $\ell(v)$ and the line connecting the origin to $\boldsymbol{u}(x)$, provided that they intersect. Clearly, $\boldsymbol{v}(x)=\boldsymbol{w}(x)$ for $x=x_{1}, x_{2}, x_{3}$, and $\boldsymbol{u}(x)$ and $\boldsymbol{w}(x)$ have the same slope for every $x$. The transformation $\boldsymbol{u}(x) \rightarrow \boldsymbol{w}(x)$ is a projective transformation from $\ell(u)$ to $\ell(v)$, therefore, it preserves the cross ratio of any four points. That is, for any $x$, we have

$$
\left(\boldsymbol{u}\left(x_{1}\right), \boldsymbol{u}\left(x_{2}\right) ; \boldsymbol{u}\left(x_{3}\right), \boldsymbol{u}(x)\right)=\left(\boldsymbol{w}\left(x_{1}\right), \boldsymbol{w}\left(x_{2}\right) ; \boldsymbol{w}\left(x_{3}\right), \boldsymbol{w}(x)\right) .
$$

Since both $\boldsymbol{u}(x)$ and $\boldsymbol{v}(x)$ are linear functions, we also have

$$
\left(\boldsymbol{u}\left(x_{1}\right), \boldsymbol{u}\left(x_{2}\right) ; \boldsymbol{u}\left(x_{3}\right), \boldsymbol{u}(x)\right)=\left(\boldsymbol{v}\left(x_{1}\right), \boldsymbol{v}\left(x_{2}\right) ; \boldsymbol{v}\left(x_{3}\right), \boldsymbol{v}(x)\right) .
$$

Hence, we can conclude that $\boldsymbol{v}(x)=\boldsymbol{w}(x)$ for all $x$. However, this is impossible, unless $\ell(u)$ and $\ell(v)$ are parallel. Indeed, suppose that $\ell(u)$ and $\ell(v)$ are not parallel, and set $x$ in such a way that $\boldsymbol{u}(x)$ is parallel to $\ell(v)$. Then $\boldsymbol{w}(x)$ cannot have the same slope as $\boldsymbol{u}(x)$, a contradiction.

Suppose that $\varepsilon$ is 2-good and let us fix it. As above, let $u_{0}^{\varepsilon}(x)$ be the position of $u_{0}$ obtained by ModifiedProcedure $\left(\bar{G}, C, u_{0}, u_{1}, x, \varepsilon\right)$, and let $\ell^{\varepsilon}$ be the line determined by $u_{0}^{\varepsilon}(x)$.

Suppose also that there exist two independent vertices of $\bar{G}, u, u^{\prime} \neq u_{0}$, such that the line determined by $\boldsymbol{u} \boldsymbol{u}^{\prime}(x)$ is parallel to $\ell^{\varepsilon}$. Then we say that $\varepsilon$ is 3 -bad for $\bar{G}$. If $\varepsilon$ is 2-good and it is not 3-bad for $\bar{G}$, then we say that it is 3-good for $\bar{G}$.

It is easy to see that, for any $0<\varepsilon, \delta<1 / 100, \ell^{\varepsilon}$ and $\ell^{\delta}$ are not parallel, therefore, for any fixed $u, u^{\prime}$, there is at most one value of $\varepsilon$ for which the line determined by $\boldsymbol{u} \boldsymbol{u}^{\prime}(x)$ is parallel to $\ell^{\varepsilon}$. Thus, with finitely many exceptions, all values $0<\varepsilon<1 / 100$ are 3-good. 
Summarizing, we have obtained the following.

Claim 8. Suppose that $\varepsilon$ is 3-good for $\bar{G}$. With finitely many exceptions, for every value of $x$, ModifiedProcedure $\left(\bar{G}, C, u_{0}, u_{1}, x, \varepsilon\right)$ gives a proper drawing of $G^{1}$.

Now we are in a position to complete the proof of Theorem 1. Proceed with each of the components as described above for $G^{1}$. For any fixed $i$, let $u_{0}^{i} v_{0}^{i}$ be the edge deleted from $G^{i}$, and denote the resulting graphs by $\bar{G}^{1}, \ldots, \bar{G}^{m}$. Let $0<\varepsilon<1 / 100$ be fixed in such a way that $\varepsilon$ is 3 -good for all graphs $\bar{G}^{1}, \ldots, \bar{G}^{m}$. This can be achieved, in view of the fact that there are only finitely many values of $\varepsilon$ which are not 3-good. Perform ModifiedProcedure $\left(\bar{G}^{i}, C^{i}, u_{0}^{i}, u_{1}^{i}, x^{i}, \varepsilon\right)$. Now the line $\ell^{i}$ determined by all possible locations of $u_{0}^{i}$ does not pass through $t_{0}^{i}$.

Note that when ModifiedProcedure $\left(\bar{G}^{i}, C^{i}, u_{0}^{i}, u_{1}^{i}, x^{i}, \varepsilon\right)$ is executed, then apart from edges with basic slopes, we use an edge with slope $r \pi / 5 \pm \varepsilon$, for some integer $r$ $\bmod 5$. By using rotations through $\pi / 5$ and a reflection, if necessary, we can achieve that each component $\bar{G}^{i}$ is drawn using the basic slopes and one edge of slope $\varepsilon$.

It remains to set the values of $x_{i}$ and draw the missing edges $u_{0}^{i} v_{0}^{i}$. Since the line $\ell^{i}$ determined by the possible locations of $u_{0}^{i}$ does not pass through $t_{0}^{i}$, by varying the value of $x^{i}$, we can attain any slope for the missing edge $t_{0}^{i} u_{0}^{i}$, except for the slope of $\ell^{i}$. By Claim 8, with finitely many exceptions, all values of $x^{i}$ produce a proper drawing of $G^{i}$. Therefore, we can choose $x^{1}, x^{2}, \ldots, x^{m}$ so that all segments $t_{0}^{i} u_{0}^{i}$ have the same slope and every component $G^{i}$ is properly drawn using the same seven slopes. Translating the resulting drawings through suitable vectors gives a proper drawing of $G$, this completes the proof of Theorem 1 .

\section{Concluding Remarks}

In the proof of Theorem 1, the slopes we use depend on the graph $G$. However, the proof shows that one can simultaneously embed all cubic graphs using only seven fixed slopes.

It is unnecessary to use $|x| \geq 50$, in every step, we could pick any $x$, with finitely many exceptions.

It seems to be only a technical problem that we needed two extra directions in the proof of Theorem 1. We believe that one extra direction would suffice.

The most interesting problem that remains open is to decide whether the number of slopes needed for graphs of maximum degree four is bounded.

\section{References}

1. Ambrus, G., Barát, J., Hajnal, P.: The slope parameter of graphs. Acta Sci. Math.(Szeged) 72 (3-4), 875-889 (2006)

2. Barát, J., Matoušek, J., Wood, D.R.: Bounded-degree graphs have arbitrarily large geometric thickness. Electr. J. Combin. 13(1), R3, 14pp. (2006)

3. Dillencourt, M.B., Eppstein, D., Hirschberg, D.S.: Geometric thickness of complete graphs. J. Graph Algorithms Appl. 4(3), 5-17 (2000)

4. Dujmović, V., Suderman, M., Wood, D.R.: Graph drawings with few slopes. Comput. Geom. 38, 181-193 (2007) 
5. Dujmović, V., Wood, D.R.: Graph treewidth and geometric thickness parameters. In: Healy, P., Nikolov, N.S. (eds.) GD 2005. LNCS, vol. 3843, pp. 129-140. Springer, Heidelberg (2006)

6. Duncan, C.A., Eppstein, D., Kobourov, S.G.: The geometric thickness of low degree graphs. In: SoCG 2004, pp. 340-346. ACM Press, New York (2004)

7. Eppstein, D.: Separating thickness from geometric thickness. In: Pach, J. (ed.) Towards a Theory of Geometric Graphs. Contemporary Math, vol. 342, pp. 75-86. AMS, Providence (2004)

8. Fáry, I.: On straight line representation of planar graphs. Acta Univ. Szeged. Sect. Sci. Math. 11, 229-233 (1948)

9. Hutchinson, J.P., Shermer, T.C., Vince, A.: On representations of some thickness-two graphs. Comput. Geom. 13, 161-171 (1999)

10. Jamison, R.E.: Few slopes without collinearity. Discrete Math. 60, 199-206 (1986)

11. Kainen, P.C.: Thickness and coarseness of graphs. Abh. Math. Sem. Univ. Hamburg 39, 8895 (1973)

12. Keszegh, B., Pach, J., Pálvölgyi, D., Tóth, G.: Drawing cubic graphs with at most five slopes. In: Kaufmann, M., Wagner, D. (eds.) GD 2006. LNCS, vol. 4372, pp. 114-125. Springer, Heidelberg (2007)

13. Mukkamala, P., Szegedy, M.: Geometric representation of cubic graphs with four directions (manuscript, 2007)

14. Mutzel, P., Odenthal, T., Scharbrodt, M.: The thickness of graphs: a survey. Graphs Combin. 14, 59-73 (1998)

15. Pach, J., Pálvölgyi, D.: Bounded-degree graphs can have arbitrarily large slope numbers. Electr. J. Combin. 13(1), Note 1, 4pp. (2006)

16. Wade, G.A., Chu, J.-H.: Drawability of complete graphs using a minimal slope set. The Computer J. 37, 139-142 (1994) 